\title{
Design of a compact gantry for carbon-ion beam therapy
}

\author{
J. Kim๑ and M. Yoon๑* \\ Department of Physics, Pohang University of Science and Technology (POSTECH), \\ Pohang, Gyeongbuk 37673, Korea
}

(Received 23 June 2019; published 4 October 2019)

\begin{abstract}
This paper presents the design of a compact gantry that uses superconducting bending magnets (BMs), for use in carbon-ion beam therapy. The size of the gantry is comparable to those of existing gantries that are used for proton-beam therapy. The designed gantry provides point-to-parallel scanning over an area of $20 \mathrm{~cm} \times 20 \mathrm{~cm}$ at the isocenter, and has rotationally invariant optics, which are enabled by quadrupole and dipole magnets together with a $90^{\circ}$ combined-function magnet with $18.6-\mathrm{cm}$ bore radius. A $90^{\circ} \mathrm{BM}$ accommodates large scanning angles; it also provides equal focusing in horizontal and vertical planes, and zero-integrated nonlinear fields to minimize beam distortion at the isocenter. Three-dimensional field analysis of the magnet, and particle-tracking simulation, validate the beam optics of the gantry and point-toparallel scanning. The Taylor map and the Lie map are shown to be useful in the analysis of magnetic fields and in optimizing the coil windings.
\end{abstract}

DOI: 10.1103/PhysRevAccelBeams.22.101601

\section{INTRODUCTION}

Carbon-ion radiotherapy (CIRT) is a favorable method to kill deeply situated tumor cells by exploiting the physical properties and biological effects of a beam of carbon nuclei $\left(\mathrm{C}^{6+}\right.$ ion beam) [1]. The Heavy-Ion Medical Accelerator in Chiba was the world's first CIRT facility; it has treated $>10,000$ patients [2], and their good prognosis led to construction of additional CIRT facilities worldwide.

The precision of treatment in a CIRT can be improved by using a gantry that can rotate $\pm 180^{\circ}$ around a patient. $\mathrm{C}^{6+}$ ion beam has large beam rigidity $(\sim 6.6 \mathrm{Tm}$ at $430 \mathrm{MeV} / \mathrm{u})$ so a gantry for CIRT must be large; as a result, it is very difficult to realize. Reducing the size of the CIRT gantry has been an important challenge in CIRT. Only two CIRT gantries are currently in operation. The first was constructed at the Heidelberg Ion Therapy Facility (HIT); the gantry has length $L=25 \mathrm{~m}$, radius $r=6.5 \mathrm{~m}$, and weight $W \sim 600$ ton. It uses normal conducting magnets. Constructing a CIRT gantry that uses only normal conducting magnets is a difficult task because of the huge size [3]. The second CIRT gantry uses superconducting technologies; it was installed at the National Institute of Radiological Sciences (NIRS) [4-6]. It has $L=13 \mathrm{~m}$, $r=5.5 \mathrm{~m}, W \sim 300$ ton, and has a maximum magnetic field $\sim 2.9$ T. To further reduce the size of CIRT gantries,

\footnotetext{
*moohyun@postech.ac.kr
}

Published by the American Physical Society under the terms of the Creative Commons Attribution 4.0 International license. Further distribution of this work must maintain attribution to the author(s) and the published article's title, journal citation, and DOI. the dipole field must be increased to reduce the bending radius.

Several preliminary studies have considered superconducting magnets with dipoles of $\sim 5 \mathrm{~T}$ for $\mathrm{C}^{6+}$ ion beam of $\sim 400 \mathrm{MeV} / \mathrm{u}$ [7-10]. The gantries can use upstream scanning or downstream scanning. Upstream scanning is performed before they encounter the final bending magnet $(\mathrm{BM})$; this method realizes orthogonal beam irradiation over the whole scanning area (parallel scanning), and is advantageous because it minimizes skin dose and simplifies treatment planning [11]. However, upstream scanning requires the final $\mathrm{BM}$ to have large aperture (bore radius $>10 \mathrm{~cm}$ ) to accommodate the scanned beam. Existing CIRT gantries use upstream scanning.

Downstream scanning is performed after they pass through the final $\mathrm{BM}$, so it does not require a large aperture. However, this method does not provide parallel scanning, and therefore requires sufficiently long space from the end of the last BM to the isocenter (source-to-axis distance), so the radius of the gantry must be increased.

Using a 130-mm bore radius 5-T superconducting combined-function magnet as a final BM [8], we could reduce the size of a CIRT gantry to $L=12 \mathrm{~m} \times r=6 \mathrm{~m}$ $[8,9]$, which is as compact as existing gantries for use in proton-beam therapy. With parallel scanning, this gantry can cover a $15 \mathrm{~cm} \times 15 \mathrm{~cm}$ scanning area at the isocenter. Although this size meets the minimum required scanning area [7,12], existing CIRT gantries have a scanning area of $20 \mathrm{~cm} \times 20 \mathrm{~cm}[3,4]$, so a gantry that is more compact and has larger scanning area than existing gantries is desirable. A suggested very compact CIRT gantry with $L=5 \mathrm{~m}$ and $r=4$ m consists of only three superconducting combinedfunction magnets of $\sim 5 \mathrm{~T}[10]$. It uses downstream scanning, 
so no large-aperture magnets are used. Its reduced size is encouraging but, to our knowledge, further studies on size of scanning area and quality of beam profiles on the scanning area (e.g., beam distortion at the isocenter) have not been presented.

In this paper, we present a compact CIRT gantry that covers $20 \mathrm{~cm} \times 20 \mathrm{~cm}$ scanning area with parallel scanning and rotation-invariant beam sizes. These abilities are realized by using a superconducting 5-T combined-function magnet that has a bore radius of $18.6 \mathrm{~cm}$. Section II introduces the layout and linear beam optics of the designed CIRT gantry. Section III shows specifications and considerations of the large aperture superconducting 5-T combined-function magnet. Section IV describes results of three-dimensional (3D) field analysis for the large aperture magnet and beam profiles obtained by particle-tracking simulation. Section V presents a conclusion.

\section{LINEAR OPTICS}

The proposed compact CIRT gantry (Fig. 1) has $L=$ $9.74 \mathrm{~m}$ and $r=3.17 \mathrm{~m}$, so its volume $\pi L r^{2} \sim 300 \mathrm{~m}^{3}$. This gantry follows optics constraints [13], which is a design to make an isocentric gantry that can transport a nonsymmetric beam without using a rotator. The gantry consists of six quadrupoles, two $45^{\circ} \mathrm{BMs}$, scanning magnets, and a $90^{\circ} \mathrm{BM}$. Settings for all gantry magnets are fixed under gantry rotation. Although beam size changes at the gantry entrance and thereafter as the gantry rotates, beam size at the gantry isocenter is invariant. Reference energy of $\mathrm{C}^{6+}$ ion beam for the gantry is $400 \mathrm{MeV} / \mathrm{u}$, which corresponds to beam rigidity of $6.345 \mathrm{Tm}$. The gantry transports an asymmetric beam of that has standard deviation $\sigma_{x}=2 \mathrm{~mm}$ in the horizontal direction and $\sigma_{y}=10 \mathrm{~mm}$ in the vertical direction to yield a round beam of $\sigma_{x}=\sigma_{y}=1.7 \mathrm{~mm}$ at the isocenter. This standard deviation corresponds to a full width at half maximum $(\mathrm{FWHM})=4 \mathrm{~mm}$, which is a typical size of a 400-MeV/u carbon beam. Twiss and beam parameters at the gantry entrance (Table I) vary along the gantry beam line (Fig. 2). All quadrupole magnets (QMs) are

TABLE I. Twiss parameters and beam parameters at the gantry entrance.

\begin{tabular}{lcc}
\hline \hline Parameter & Value & Unit \\
\hline Horizontal emittance $\left(\varepsilon_{x}\right)$ & 1 & $\pi \mathrm{mm}$ mrad \\
Vertical emittance $\left(\varepsilon_{y}\right)$ & 5 & $\pi \mathrm{mm} \mathrm{mrad}$ \\
Horizontal beta function $\left(\beta_{x}\right)$ & 4 & $\mathrm{~m}$ \\
Vertical beta function $\left(\beta_{y}\right)$ & 20 & $\mathrm{~m}$ \\
Horizontal alpha function $\left(\alpha_{x}\right)$ & 0 & $\ldots$ \\
Vertical alpha function $\left(\alpha_{y}\right)$ & 0 & $\ldots$ \\
Horizontal rms beam size $\left(\sigma_{x}\right)$ & 2 & $\mathrm{~mm}$ \\
Vertical rms beam size $\left(\sigma_{y}\right)$ & 10 & $\mathrm{~mm}$ \\
Root-mean-square momentum & 0.002 & $\ldots$ \\
$\quad$ spread $\left(\sigma_{\Delta p / p_{0}}\right)$ & & \\
\hline \hline
\end{tabular}

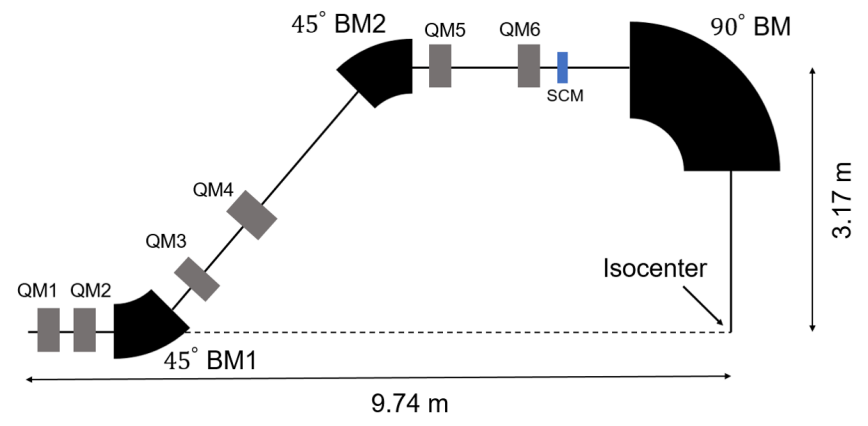

FIG. 1. Layout of the CIRT gantry. QM: quadrupole magnet; BM: bending magnet. The radius from the rotational axis to the beam axis is $3.17 \mathrm{~m}$. The radius from the rotational axis to the outer part of the iron shielding is $3.83 \mathrm{~m}$.

normal conducting magnets. Two $45^{\circ} \mathrm{BMs}$ (Table II) are small-aperture superconducting magnets with 5-T dipole field. The $90^{\circ} \mathrm{BM}$ (Table III) is a large-aperture superconducting combined-function magnet with 5-T dipole field. Magnetic elements except the $90^{\circ}$ magnet are assumed to follow the hard-edge model. The $90^{\circ}$ magnet has an important function, so we designed a preliminary threedimensional (3D) model by using OPERA3D [14], then used calculated field data from the model to match linear beam optics. Specifications and analysis for the $90^{\circ}$ magnet are presented in subsequent sections.

When a linear matrix from the entrance to the isocenter is represented as $R_{i j}$, with beams phase-space coordinates $\left[x, x^{\prime}, y, y^{\prime}, l, \delta\left(=\Delta p / p_{0}\right)\right]$, rotational invariant beam optics of the gantry is achieved by $R_{11}=R_{33}=0$, doubleachromatic beam optics is achieved by $R_{16}=R_{26}=0$, and the size of round beam is controlled by $R_{12}\left(=R_{34}\right)$. Here, the value for $R_{12}$ is set to $3.4 \mathrm{~m} / \mathrm{rad}$ to yield a round Gaussian beam with FWHM $=4 \mathrm{~mm}$ at the isocenter. Horizontal and vertical root-mean-square (rms) beam envelopes along the gantry with respect to rotation angle clearly show beam optics matched well (Fig. 3). As the

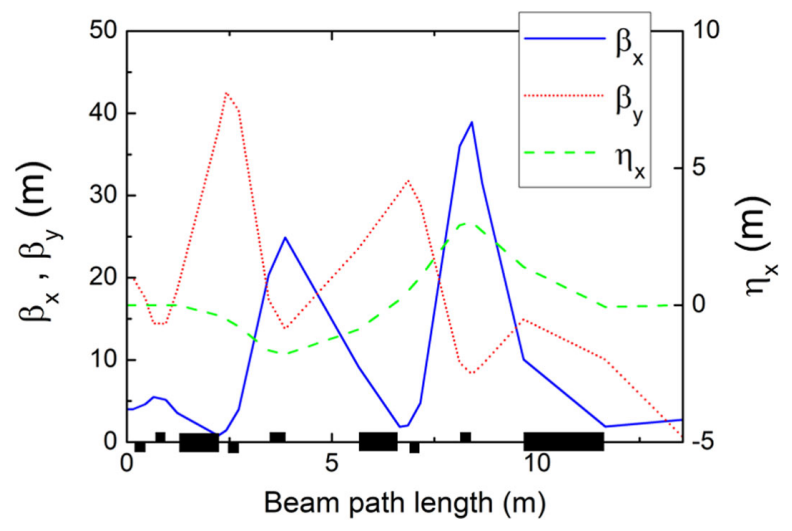

FIG. 2. Twiss parameters along the gantry beam line (without rotation; 0 degree from the vertical position). Blue curve: horizontal beta function; red curve: vertical beta function; green curve: horizontal dispersion function. 
TABLE II. Parameters for normal conducting quadrupole magnets.

\begin{tabular}{lccccccc}
\hline \hline Name & QM1 & QM2 & QM3 & QM4 & QM5 & QM6 & Unit \\
\hline Length & 0.30 & 0.30 & 0.30 & 0.40 & 0.30 & 0.30 & $\mathrm{~m}$ \\
$\begin{array}{l}\text { Normalized field } \\
\text { gradient (K1) }\end{array}$ & -1.454 & 3.360 & -2.466 & 2.367 & -1.811 & 3.559 & $1 / \mathrm{m}^{2}$ \\
Half aperture & 36.0 & 36.0 & 46.5 & 60.0 & 46.5 & 36.0 & $\mathrm{~mm}$ \\
Pole-tip field & 0.332 & 0.768 & 0.727 & 0.901 & 0.534 & 0.813 & $\mathrm{~T}$ \\
\hline \hline
\end{tabular}

TABLE III. Parameters for superconducting $45^{\circ}$ bending magnets.

\begin{tabular}{lccc}
\hline \hline Name & $45^{\circ} \mathrm{BM} 1$ & $45^{\circ} \mathrm{BM} 2$ & Unit \\
\hline Bending angle & -45 & 45 & $\mathrm{deg}$ \\
Bending radius & 1.269 & 1.269 & $\mathrm{~m}$ \\
Half aperture & 46.5 & 60.0 & $\mathrm{~mm}$ \\
Dipole field & 5 & 5 & $\mathrm{~T}$ \\
\hline \hline
\end{tabular}

rotation angle varies, the size of the beam changes while it passes through magnetic elements, but it converges to a fixed value at the isocenter. For proper control of the input beam size in practical operation, collimators are needed just before the gantry entrance.

For two-dimensional (2D) scanning on the transverse plane at the isocenter, parallel scanning is adopted. This method is realized by using a single scanning magnet located upstream of the $90^{\circ}$ magnet (Fig. 1). The scanning magnet performs horizontal and vertical scanning to cover a $20 \mathrm{~cm} \times 20 \mathrm{~cm}$ area at the isocenter. The positions of scanning magnets are not separated for horizontal and vertical planes, as in other CIRT gantries $[3,4]$. From the position of the scanning magnet to the isocenter, point-toparallel optics is satisfied for both horizontal and vertical plane $\left(R_{22}=R_{44}=0\right)$.

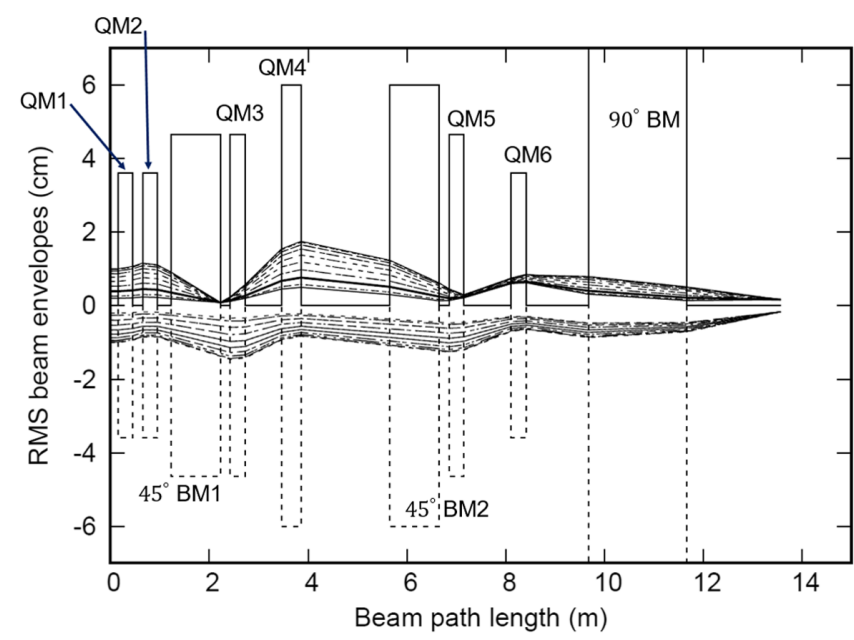

FIG. 3. Root-mean-square beam envelopes with respect to the rotation angle at the gantry entrance. Upper curves show horizontal envelopes; lower curves show vertical envelopes, as a function of rotation angles up to $\pm 180^{\circ}$.

\section{THE $90^{\circ}$ BENDING MAGNET}

In this section, we present specifications and considerations of the large-aperture superconducting $90^{\circ}$ combinedfunction magnet (Figs. 4,5; Tables IV, V). The magnet is toroidal with a bending radius of $1.269 \mathrm{~m}$ and a bore radius of $18.6 \mathrm{~cm}$. A 40-cm-thick toroid of iron is wrapped around the coil to reduce the stray field and strengthen the magnetic field inside the coil aperture. For field calculation inside the shielding iron, the hysteresis data of 1010 steel is used [14].

For coil winding, we used double-helical canted-cosinetheta (CCT) winding in toroidal coordinates [Fig. 4(a)] $[7,8,15,16]$; this arrangement generates perpendicular (a)

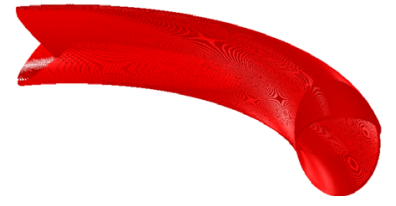

(b)

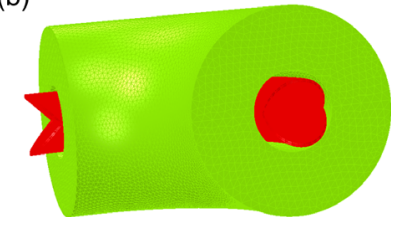

FIG. 4. 3D view of the coil winding (a) and shielding iron (b) of the superconducting $90^{\circ}$ combined-function magnet.

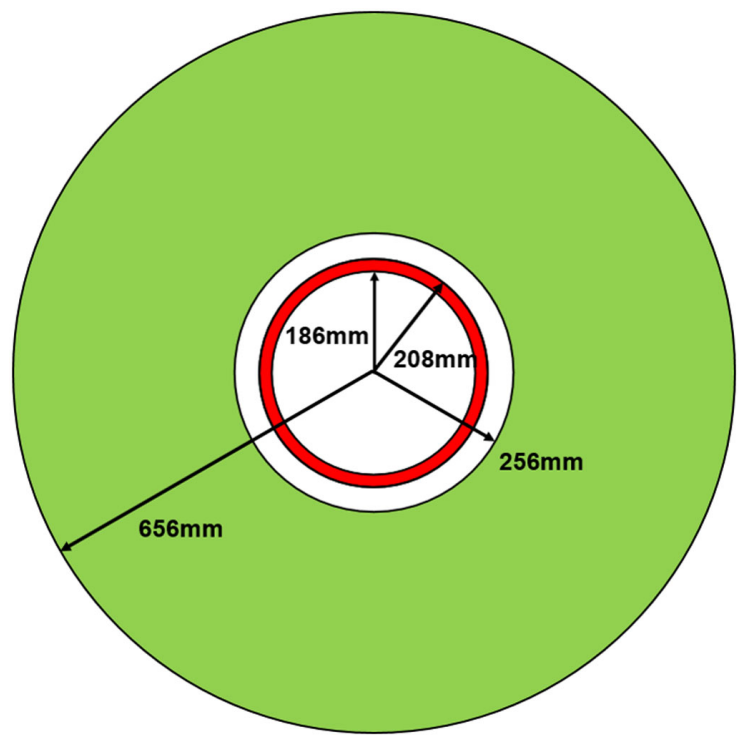

FIG. 5. Cross-sectional view of the superconducting $90^{\circ}$ combined-function magnet. Red: coil; green: shielding iron. 
TABLE IV. Geometric parameters of the superconducting $90^{\circ}$ combined-function magnet.

\begin{tabular}{lcc}
\hline \hline Parameter & Value & Unit \\
\hline Bending radius & 1.269 & $\mathrm{~m}$ \\
Inner radius of coil & 186 & $\mathrm{~mm}$ \\
Outer radius of coil & 208 & $\mathrm{~mm}$ \\
Inner radius of shielding iron & 256 & $\mathrm{~mm}$ \\
Outer radius of shielding iron & 656 & $\mathrm{~mm}$ \\
Number of coil layers & 2 & $\ldots$ \\
Number of coil winding for $2 \pi$ torus & 2080 & $\ldots$ \\
Width of coil & 0.8 & $\mathrm{~mm}$ \\
Thickness of coil & 22 & $\mathrm{~mm}$ \\
\hline \hline
\end{tabular}

TABLE V. Magnetic parameters of the superconducting $90^{\circ}$ combined-function magnet.

\begin{tabular}{lccc}
\hline \hline Parameter & Symbol & Value & Unit \\
\hline Dipole field & $B_{0}$ & 5 & $\mathrm{~T}$ \\
Quadrupole field & $B_{1}$ & -2.012 & $\mathrm{~T} / \mathrm{m}$ \\
Integrated sextupole field & $\left|\int B_{2} d l\right|$ & $<0.1$ & $\mathrm{~T} / \mathrm{m}$ \\
Integrated octupole field & $\left|\int B_{3} d l\right|$ & $<1$ & $\mathrm{~T} / \mathrm{m}^{2}$ \\
Integrated field quality & $\mid \int \Delta B L /$ & $\leq 1.2 \times 10^{-3}$ & $\ldots$ \\
& $\int B L \mid$ & & \\
Maximum field at coil & $\ldots$ & 6.3 & $\mathrm{~T}$ \\
Current density & $\ldots$ & 559 & $\mathrm{~A} / \mathrm{mm}^{2}$ \\
Total stored energy & $\ldots$ & 2.93 & $\mathrm{MJ}$ \\
\hline \hline
\end{tabular}

multipole-field components of arbitrary order inside the coil aperture. Here we briefly introduce the geometry and characteristics of the method.

Orthogonal variables for the toroidal coordinate system are $(R, \phi, \theta): R$ is the minor radius of a torus, $\phi$ is the toroidal angle, and $\theta$ is the polar angle. In the toroidal coordinate system, the coil winding for the double-helical CCT winding is described by [7]

$\phi=\frac{\theta}{n}+a_{0} \sin \theta+a_{1} \sin 2 \theta+a_{2} \sin 3 \theta+a_{3} \sin 4 \theta+\cdots$,

where $n$ is the number of winding turns on a $2 \pi$ torus, and winding coefficients, $a_{0}, a_{1}, a_{2}, a_{3}$, control multipole field components: dipoles, quadrupoles, sextupoles, octupoles, and upward. Each coefficient generates a pure multipole field of corresponding order in free space.

Parameters of the $90^{\circ}$ combined-function magnet are determined to satisfy the following considerations.

\section{A. Good field region of $20 \mathrm{~cm} \times 20 \mathrm{~cm}$}

This design assumes a scanning area of $20 \mathrm{~cm} \times 20 \mathrm{~cm}$. To accommodate the scanned beam, the size of the good field region must be as large as the size of the scanning area.
For a single-pass magnetic element, required integrated field quality over the good field region should be less than $\sim 10^{-3}$. The coil's bore radius of $18.6 \mathrm{~cm}$ is determined so that the good field region includes a larger area than the scanning area of $20 \mathrm{~cm} \times 20 \mathrm{~cm}$ (or circumscribed circle of $14.4 \mathrm{~cm}$ radius over $20 \mathrm{~cm} \times 20 \mathrm{~cm}$ ). Estimation was based on a previous study that describes the ratio of good field regions radius and bore radius for the CCT winding method is approximately $85 \%$ [7].

\section{B. Equal focusing for horizontal and vertical planes}

With a 5-T dipole field at the central region, quadrupole strength was calculated to meet equal focusing for horizontal and vertical planes from the scanning magnet to the isocenter. Under this condition, linear matrices for horizontal and vertical planes are the same $\left(R_{11}=R_{33}, R_{12}=\right.$ $R_{34}, R_{21}=R_{43}, R_{22}=R_{44}$ ). This beam optics corresponds to that of a hard-edge combined-function magnet that has a field index of 0.5. From this condition, point-to-parallel optics for both horizontal and vertical planes from the scanning magnet to the isocenter can be achieved. The beam optics of the $90^{\circ}$ magnet does not have a hard edge, but includes a fringe field distribution, so several attempts have been made to find the correct quadrupole strength that satisfies this condition.

We set this condition as one of the ways to reduce the beam distortion at the scanning area although this is not an essential requirement for an isocentric gantry. Specifically, when parallel scanning is performed through a $90^{\circ} \mathrm{com}-$ bined-function magnet of $1.269-\mathrm{m}$ bending radius, the scanning angle required to reach the edge of the $20 \mathrm{~cm} \times$ $20 \mathrm{~cm}$ area at the isocenter is estimated to be $\sim 50 \mathrm{mrad}$, which is more than twice the maximum steering angle of the NIRS gantry [4]. The degree of beam distortion increases nonlinearly as scanning angle increases [7], so $50 \mathrm{mrad}$ is already very large. Nevertheless, if maximum scanning angles that reach the edge of the scanning area are biased in the vertical plane, like $40 \mathrm{mrad}$ for the horizontal edge and $60 \mathrm{mrad}$ for the vertical edge, distortion of the beam shape on the vertical edge can be severe. This possibility can be avoided by adopting beam optics that has equal focusing strength for horizontal and vertical planes.

\section{Zero-integrated fields for both sextupole and octupole}

Nonlinear field components are regulated for the $90^{\circ}$ magnet to minimize beam distortion at the isocenter. Considering large scanning angles $\sim 50 \mathrm{mrad}$ to cover the whole $20 \mathrm{~cm} \times 20 \mathrm{~cm}$ scanning area, the sextupole components and octupole components are calculated to achieve zero-integrated fields along the magnet. Tolerances were set to $0.1 \mathrm{~T} / \mathrm{m}$ for the integrated sextupole field, and $1 \mathrm{~T} / \mathrm{m}^{2}$ for the integrated octupole field. 
To achieve zero-integrated fields for the sextupole and the octupole, we concentrate only on making a zerosextupole field and zero-octupole field on the central region. We make this choice because end harmonics produced by the CCT winding are canceled around magnet ends. This characteristic was first explained theoretically in [16] and presented for a straight dipole magnet [17] and a QM [18] made using the CCT winding method. Although our $90^{\circ}$ magnet is a BM with partially toroidal shape, we expect this characteristic to hold. This regulation can be achieved naturally for the CCT coil winding in free space without using the corresponding coefficients $a_{2}, a_{3}$ in Eq. (1), because $a_{2}=a_{3}=0$. However, the magnet model includes shielding iron, and magnetic shielding can create a nonlinear field distribution inside the coil aperture. Therefore, $a_{2}$ and $a_{3}$ must be optimized to correct nonzero nonlinear fields.

\section{FIELD ANALYSIS}

The 3D field of the $90^{\circ}$ magnet was obtained by simulation using OPERA3D [14], and several kinds of field analysis were conducted. Along the midplane of the $90^{\circ}$ magnet, the field distribution has a 5-T dipole field at the center and a field gradient inside the coil aperture (Fig. 6). The field saturated at $\sim 2 \mathrm{~T}$ near the shielding iron, and the field was cut off outside of the shielding iron.

Maximum field strength at the coil region was found to be $6.3 \mathrm{~T}$. At this field strength, Refs. $[19,20]$ show that NbTi strands at $4.2 \mathrm{~K}$ have a critical current of $1950 \mathrm{~A} / \mathrm{mm}^{2}$. For NbTi cable with a copper-to-superconductor ratio of $1.5: 1$, critical current is approximately $800 \mathrm{~A} / \mathrm{mm}^{2}[21,22]$. Hence, our $90^{\circ}$ magnet whose current density is $559 \mathrm{~A} / \mathrm{mm}^{2}$ can be operated with $\sim 30 \%$ critical current margin.

Integrated field quality was calculated over a $20 \mathrm{~cm} \times$ $20 \mathrm{~cm}$ square region (Fig. 7). The paths along which the integration was performed are not real particle paths but geometrically parallel paths that are sufficiently long to include the fringe field region of the magnet. The integrated

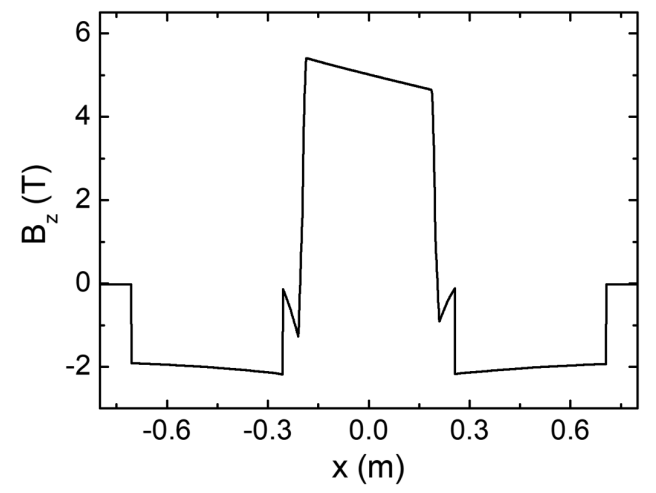

FIG. 6. Magnetic field distribution along the midplane of the superconducting $90^{\circ}$ combined-function magnet. The origin of the $\mathrm{x}$ coordinates is the center of the magnet bore.

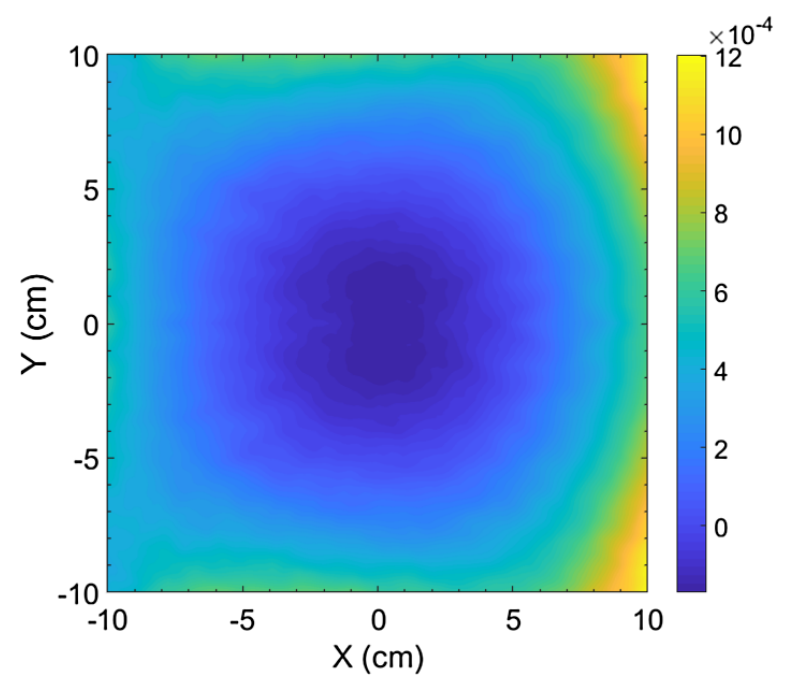

FIG. 7. Integrated field quality of the superconducting $90^{\circ}$ combined-function magnet.

field quality within the $20 \mathrm{~cm} \times 20 \mathrm{~cm}$ region was $\leq 1.2 \times 10^{-3}$.

To visualize the distribution of end harmonics, we examined multipole coefficients along the reference path of the $90^{\circ}$ magnet (Fig. 8). The dipole and quadrupole components decreased continuously around the fringe field region and the combined sinusoidal waves of the sextupole and octupole. Specifically, for a sextupole field, the distribution is antisymmetric with respect to the physical end of the magnet; therefore, the end harmonic is canceled well. The calculated integrated sextupole field along the magnet was $-0.088 \mathrm{~T} / \mathrm{m}$. The octupole field was not symmetrical, but numerical integration along the distribution showed that the area of the positive region and the area of the negative region are almost equal. The calculated integrated octupole field along the magnet was $0.802 \mathrm{~T} / \mathrm{m}^{2}$. These values for the integrated sextupole and integrated octupole satisfy the requirements presented in Sec. III.

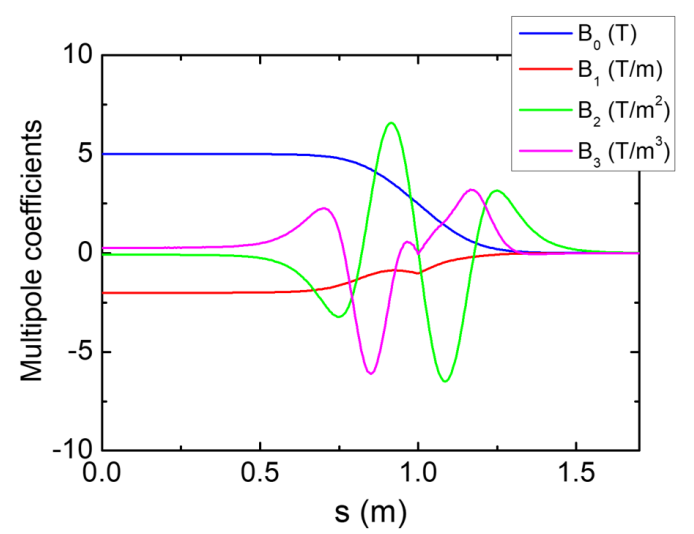

FIG. 8. Multipole field distributions along the reference path of the superconducting $90^{\circ}$ combined-function magnet (half view). 


\section{PARTICLE TRACKING}

We performed numerical particle tracking to validate beam optics of the compact gantry. Linear matrices in the hard-edge model are used for beam transport before the scanning magnets, where $45^{\circ}$ bending magnets, QMs and drift spaces are aligned in order. Then 3D field data of the final bend were used from the scanning magnet to the isocenter. For numerical integration of Lorentz equation with the 3D field data, we used OPERA3D postprocessor [14], which uses a Runge-Kutta integrator.

To visualize point-to-parallel optics, particles were launched with distributed scanning angles from the scanning magnet (Fig. 9). The particles moved toward the isocenter in parallel direction regardless of scanning angle.

We obtained transverse linear matrices from the scanning magnet to the isocenter by least-square minimization of tracking results of 17 prepared particles [23]:

$$
\begin{aligned}
& R_{h}=\left(\begin{array}{cc}
-0.421 \mathrm{~m} / \mathrm{m} & 2.099 \mathrm{~m} / \mathrm{rad} \\
-0.477 \mathrm{rad} / \mathrm{m} & 0.003 \mathrm{rad} / \mathrm{rad}
\end{array}\right), \\
& R_{v}=\left(\begin{array}{cc}
-0.416 \mathrm{~m} / \mathrm{m} & 2.112 \mathrm{~m} / \mathrm{rad} \\
-0.475 \mathrm{rad} / \mathrm{m} & 0.006 \mathrm{rad} / \mathrm{rad}
\end{array}\right),
\end{aligned}
$$

where $R_{h}$ is the linear matrix for the horizontal plane and $R_{v}$ is the linear matrix for the vertical plane. This result shows that equal focusing for horizontal and vertical planes is achieved for the $90^{\circ}$ magnet $\left(R_{h} \cong R_{v}\right)$, and that to reach the edge of the $20 \mathrm{~cm} \times 20 \mathrm{~cm}$ scanning area at the isocenter by parallel scanning, point-to-parallel optics $\left(R_{22}=R_{44} \cong 0\right)$ and scanning angles of $\sim 50 \mathrm{mrad}$ are required $\left(R_{12}=R_{34} \cong 2.1\right)$.

\section{A. Taylor map}

For fast multiparticle tracking simulation, we mainly used a Taylor map from the scanning magnet to the isocenter, after using Runge-Kutta integration to confirm equivalence. From the particle tracking data we extracted a Taylor map,

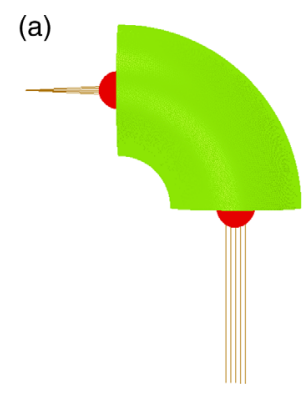

(b)

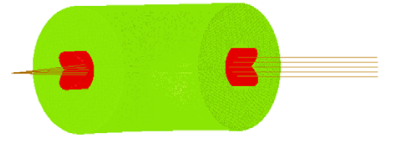

FIG. 9. Parallel scanning for horizontal plane (a) and vertical plane (b).

$$
\begin{aligned}
X_{i, \mathrm{out}}= & \sum_{i}^{6} R_{i j} X_{j, 0}+\sum_{j \leq k}^{6} T_{i j k} X_{j, 0} X_{k, 0} \\
& +\sum_{j \leq k \leq l}^{6} U_{i j k l} X_{j, 0} X_{k, 0} X_{l, 0}+\cdots,
\end{aligned}
$$

by least-square minimization using beam coordinates of a bunch of particles at the scanning magnets (initial coordinates) and at the isocenter (final coordinates) which were tracked using the Runge-Kutta integration method. To assess the equivalence of the two tracking methods, we first compared results of the single-particle tracking simulation. A total of 121 reference particles were launched at angles of 0 to $50 \mathrm{mrad}$ in increments of $12.5 \mathrm{mrad}$ at the scanning position. Tracking results are compared with firstorder, second-order, and third-order Taylor maps (Fig. 10). Better agreement is seen as $(X, Y)$ is close to the origin and order of a Taylor map is increased. At $(10 \mathrm{~cm}, \pm 10 \mathrm{~cm})$, maximal position differences $\left(\sqrt{\Delta X^{2}+\Delta Y^{2}}\right)$ between the Runge-Kutta integration and the first, second, and thirdorder Taylor maps are 5.12, 1.25, and $0.82 \mathrm{~mm}$, respectively. At the isocenter, the particles were almost linearly distributed over the squared scanning area without finetuned scanning angles; this result means that the nonlinear effect is canceled well. The remaining dominant nonlinear effects are explained by a second-order Taylor map, and the deviation that occurs near the edge of the scanning area is due to a third-order effect, as can be seen from the thirdorder Taylor map.

We also compared results of multiparticle tracking simulation (Fig. 11). The third-order Taylor map gives good agreement with Runge-Kutta integration; the two methods disagree noticeably only at the edge. Therefore,

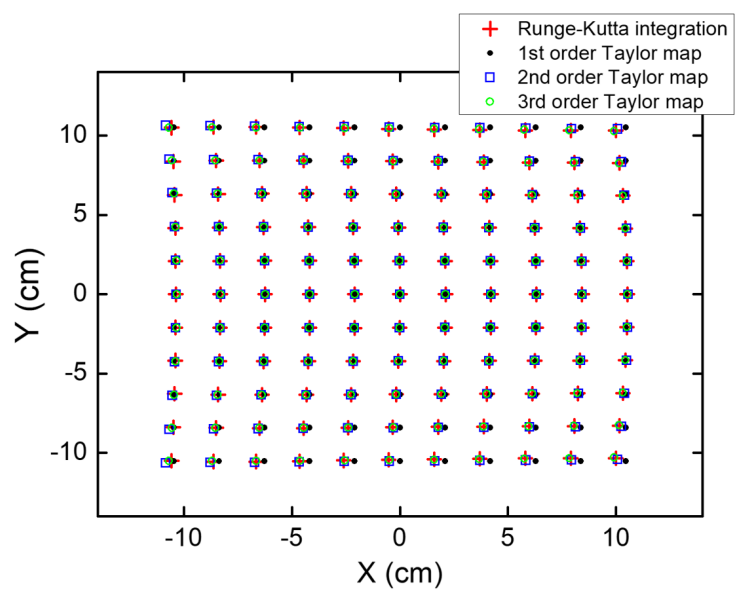

FIG. 10. Single particle distributions at the isocenter for different scanning angles with a $15 \mathrm{mrad}$ interval for both transverse planes, from 0 to $50 \mathrm{mrad}$. Colors of symbols indicate the tracking methods: red, Runge-Kutta integration; black, first-order Taylor map; blue, second-order Taylor map; green, third-order Taylor map. 


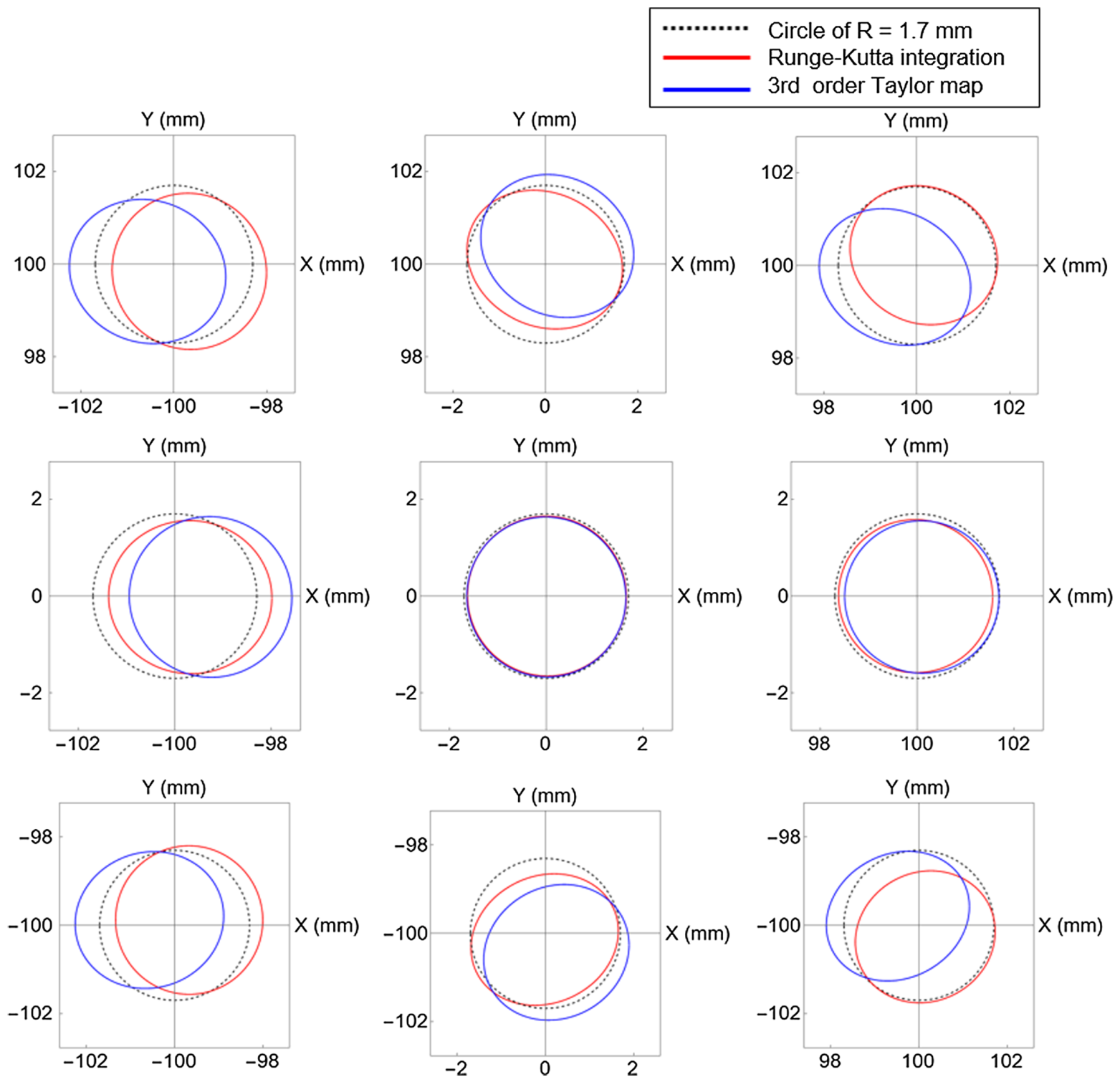

FIG. 11. 1- $\sigma$ beam ellipses of tracking results at the eight edges and the center of the scanning area. Red ellipses: Runge-Kutta integration; blue ellipses: third-order Taylor map. Each beam consists of 5000 particles. Black dashed circles with radius 1.7 mm are drawn as references.
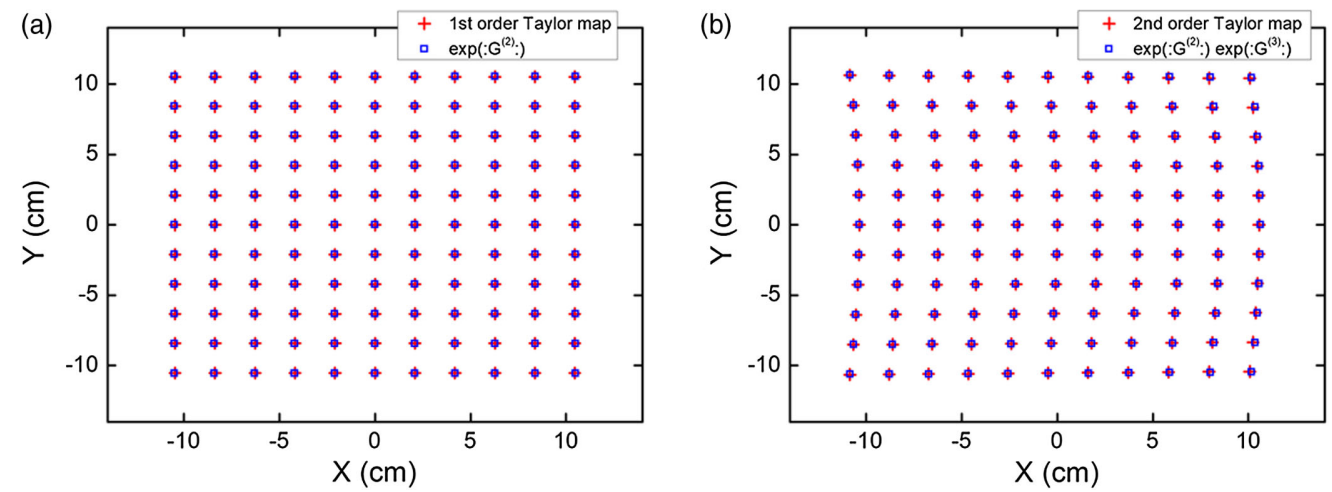

FIG. 12. Single-particle distributions at the isocenter for different scanning angles with 15-mrad interval for both transverse planes, from 0 to $50 \mathrm{mrad}$. Tracking results are drawn for first-order Taylor map and corresponding Lie map, $\exp \left(: G^{(2)}:\right)(\mathrm{a})$, and second-order Taylor map and corresponding Lie map, $\exp \left(: G^{(2)}:\right) \exp \left(: G^{(3)}:\right)(\mathrm{b})$. 

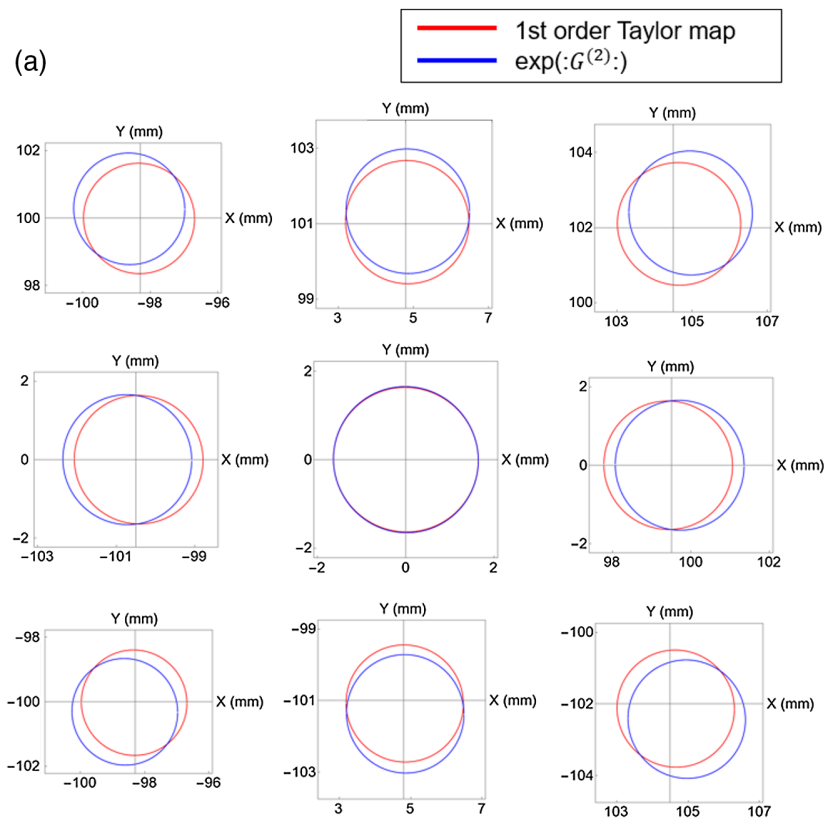

(b)

2nd order Taylor map $\exp \left(: G^{(2)}:\right) \exp \left(: G^{(3)}:\right)$
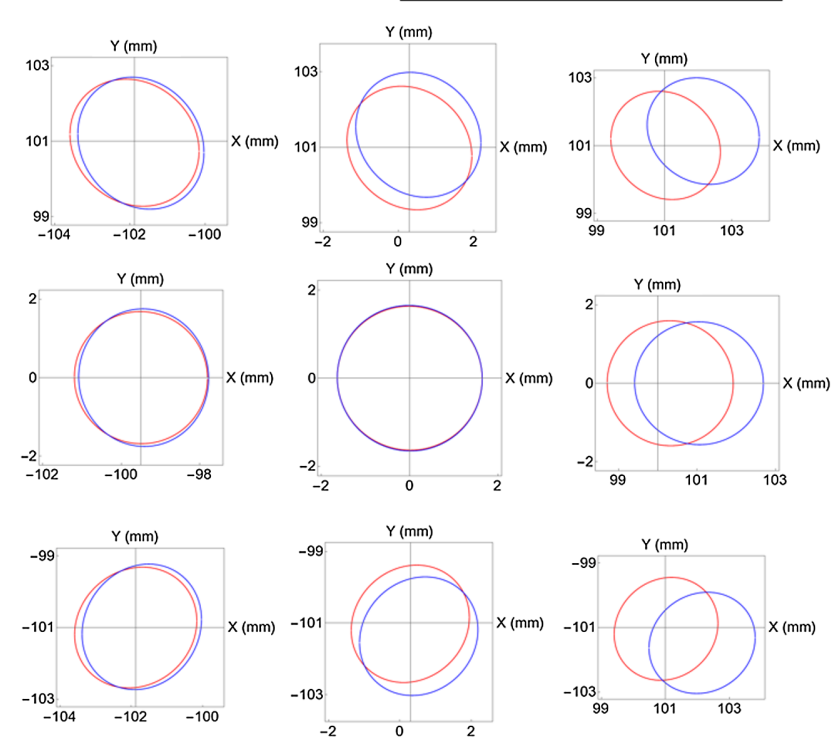

FIG. 13. 1- $\sigma$ beam ellipses at the eight edges and the center of the scanning area. Tracking results are drawn for first-order Taylor map and corresponding Lie map, $\exp \left(: G^{(2)}:\right)\left(\right.$ a), and second-order Taylor map and corresponding Lie map, $\exp \left(: G^{(2)}:\right) \exp \left(: G^{(3)}:\right)(\mathrm{b})$.

we launched eight beams with fine-tuned scanning angles for the edge and a beam for the center as a reference. Each beam consists of 5000 particles and has a Gaussian distribution (Table I). We see that centers of ellipses that represent the results of the two tracking methods differ by $<0.5 \mathrm{~mm}$, as already seen in Fig. 10 and beam shapes agree well, so we can use the third-order Taylor map method instead of the Runge-Kutta integration method.

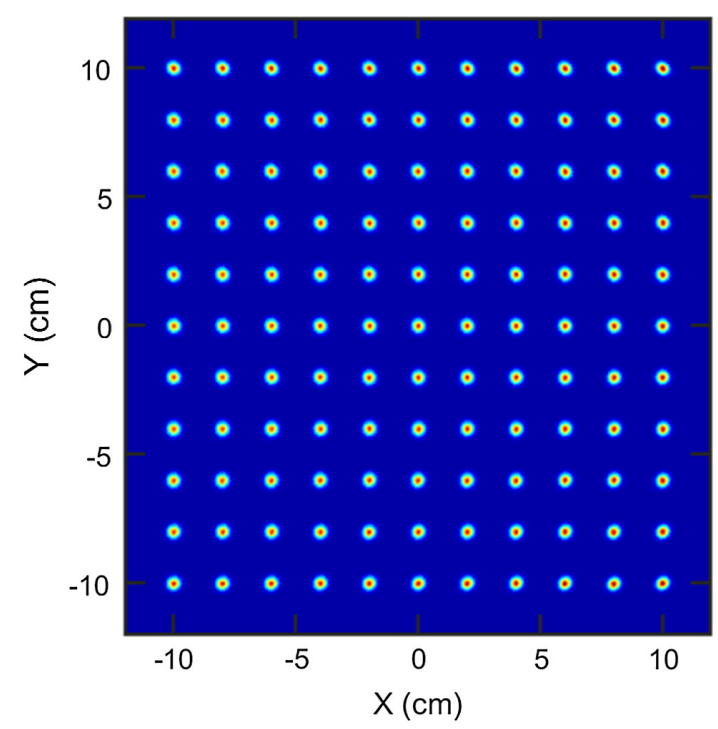

FIG. 14. Beam profiles at the isocenter. The third-order Taylor map is used for tracking simulation. Scanning angles are adjusted for aligned beam profiles. Each beam consists of 5000 particles.

\section{B. Lie map}

We also obtained a Lie map from the Taylor map. With implementation of the Dragt-Finn factorization method [24,25], corresponding Lie generators were extracted from the Taylor map, and the Lie map was constructed from Lie generators. A Lie map is intrinsically symplectic, so good agreement between a Taylor map and a Lie map implies that the Taylor map is almost symplectic. Our $90^{\circ}$ magnet has short bending radius $(\rho=1.269 \mathrm{~m})$ and large aperture $(r=0.186 \mathrm{~m})$ compared to typical magnetic elements, so this is an unusual example of symplectification.

First, beam coordinates $\left(x, x^{\prime}, y, y^{\prime}\right)$ were transformed to canonical variables $\left(x, p_{x}, y, p_{y}\right)$ [26],

$$
\begin{aligned}
& p_{x}=(1+\delta) \frac{x^{\prime}}{\sqrt{1+x^{\prime 2}+y^{\prime 2}}}, \\
& p_{y}=(1+\delta) \frac{y^{\prime}}{\sqrt{1+x^{\prime 2}+y^{\prime 2}}} .
\end{aligned}
$$

Then a factorization process was used to extract Lie generators up to corresponding orders of the Taylor map, i.e., $G^{(2)}$ for $R_{i j}, G^{(3)}$ for $T_{i j k}, G^{(4)}$ for $U_{i j k l}$. Here, $G^{(n)}$ is calculated as

$$
\begin{gathered}
G^{(2)}=-\frac{1}{2} \tilde{X} F X, \\
G^{(n)}=-\frac{1}{n} \sum_{i, j} X_{r, i}^{(n-1)} S_{i, j} X_{j},
\end{gathered}
$$



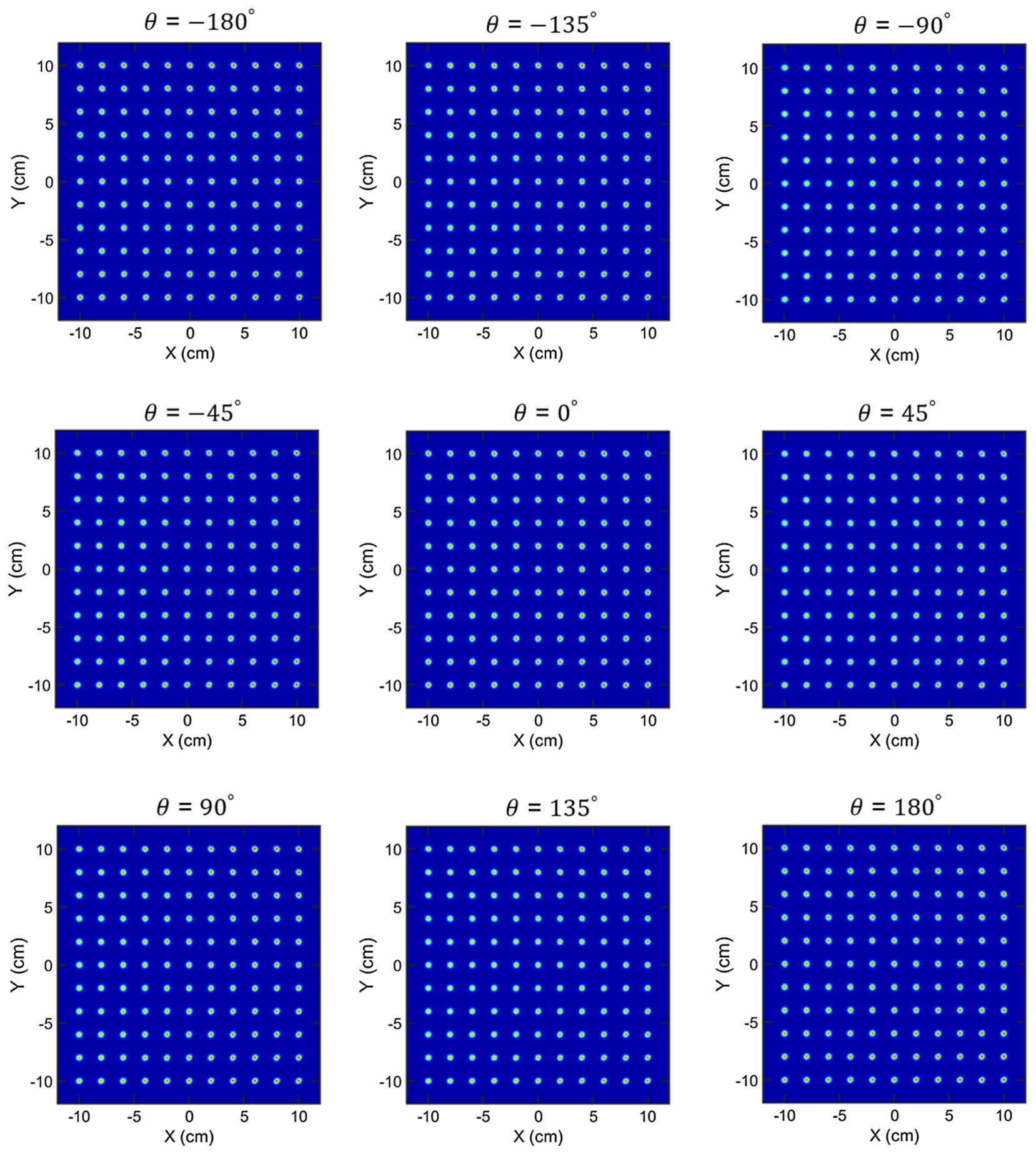

FIG. 15. Beam profiles at the isocenter for rotation angles from $-180^{\circ}$ to $+180^{\circ}$.

where $S_{i j}$ is the $6 \times 6$ asymmetric symplecticity matrix, $F$ is a symmetric matrix that satisfies $e^{: G^{(2)}}: X=e^{S F} X$, and

$$
X_{r, i}^{(n-1)}=\left(e^{-: G^{(n-1)}}: \cdots e^{-: G^{(3)}}: e^{-: G^{(2)}}: X_{i, \text { out }}\right)-X_{i, 0}
$$

is the remainder of the factorization process of corresponding order.
The resulting Lie map has the form

$$
X_{\text {out }}=M X_{i, 0}=e^{: G^{(2)}}: e^{: G^{(3)}}: e^{: G^{(4)}}: \ldots e^{: G^{(n+1)}}: X_{i, 0},
$$

where each exponential component can be represented as infinite series

$$
e^{: F:} X_{i 0}=\sum_{n=0}^{\infty} \frac{: F:^{n}}{n !} X_{i 0}
$$


To preserve the symplecticity of the map, the series expansion of numerical calculation of Eq. (9) must be truncated at sufficiently high order (i.e., $n \geq 10$ for $e^{: G^{(4)}}: X_{i 0}$ ).

We compared the Taylor map and the resulting Lie map order by order for single-particle tracking over the entire scanning field (Fig. 12) and for multiparticle tracking (5000 particles for each beam) around the edge of the scanning field (Fig. 13). The results show good agreement up to $G^{(3)}$; this result verifies the near-symplecticities of the first-order Taylor map and of the second-order Taylor map.

$X_{\text {out }}=e^{: G^{(2)}}: e^{: G^{(3)}}: e^{: G^{(4)}}: X_{i, 0}$ was not included in this work. When calculating Eqs. (8) and (9), we also avoided any kinds of approximation such as truncation or the Baker-Campbell-Hausdorff formula, because maximum variables of the map are large (i.e., $p_{x}, p_{y} \sim 0.05 \mathrm{rad}$ for initial coordinates, $x, y \sim 0.10 \mathrm{~m}$ for final coordinates). Such direct calculation increases accuracy, but also increases computing time.

A little distortion of beam shapes remained at the edges of the ellipses (Fig. 11). Although we canceled integrated fields for sextupole and octupole, the process was insufficient to obtain perfect circular beam shapes at the edge; the failure occurred because the steering angles were large. However, the target is continuously irradiated with several beams with varying scanning angles to fill the target region during radiotherapy, so the degree of distortion on our results will not be a critical problem.

Finally, multiparticle tracking simulation for the entire $20 \mathrm{~cm} \times 20 \mathrm{~cm}$ scanning area was performed using the third-order Taylor map and beam profiles were drawn (Fig. 14). Steering angles were fine-tuned to transfer each beam to an ordered position in the scanning region. Beam profiles were also drawn under various rotation angles of the gantry (Fig. 15); they confirm the rotational invariant beam optics of the compact gantry.

\section{CONCLUSION}

We presented the design of a compact gantry for carbonbeam therapy. The size of the gantry was reduced to length $9.74 \mathrm{~m}$ and radius $3.17 \mathrm{~m}$ by using 5-T superconducting bending magnets and normal conducting quadrupoles for $\mathrm{C}^{6+}$ ion beam that have energy of $400 \mathrm{MeV} / \mathrm{u}$. It can transport an asymmetric beam, and yields a beam with $\mathrm{FWHM}=4 \mathrm{~mm}$ at the isocenter regardless of rotating angle.

Upstream parallel scanning that covers a $20 \mathrm{~cm} \times 20 \mathrm{~cm}$ scanning area at the isocenter was achieved using a last $90^{\circ}$ bending magnet that has a bore radius of $18.6 \mathrm{~cm}$. To minimize nonlinear effects on the scanning area, it has equal focusing strengths for transverse planes and zerointegrated fields for sextupole and octupole. 3D field calculation and field analysis of the magnet including shielding iron were performed to verify field quality and field distribution. Particle-tracking simulation was performed using 3D field data of the last bending magnet. Taylor map coefficients and Lie generators that describe the beam optics of the scanning section were extracted. They showed that nonlinear effects were canceled well. The remaining nonlinear effects were mostly explained using second-order coefficients, then third-order coefficients provided fast mapping equivalent with usual RungeKutta integration. Extracted Lie generators up to $G^{(3)}$ and resulting Lie maps showed good agreements with corresponding Taylor maps. Tracking results of Gaussian distributed beams with various scanning angles demonstrated that beam shapes are maintained over the entire scanning area for any rotating angle.

\section{ACKNOWLEDGMENTS}

This work was supported by POSTECH Basic Science Research Institute Grant and by Ministry of Science and ICT. We would like to thank D. S. Robin at Lawrence Berkeley National Laboratory for valuable suggestions and encouraging discussions.

[1] U. Amaldi and G. Kraft, Radiotherapy with beams of carbon ions, Rep. Prog. Phys. 68, 1861 (2005).

[2] O. Mohamad, H. Makishima, and T. Kamada, Evolution of carbon ion radiotherapy at the National Institute of Radiological Sciences in Japan, Cancers 10, 66 (2018).

[3] R. Fuchs and U. Weinrich, Assembly of the carbon beam gantry at the Heidelberg Ion Therapy (HIT) accelerator, in Proceedings of the 11th European Particle Accelerator Conference, EPAC 2008, Genoa, 2008 (JACoW, Genoa, 2008), p. 1839.

[4] Y. Iwata, K. Noda, T. Shirai, T. Murakami, T. Furukawa, S. Mori, T. Fujita, A. Itano, K. Shouda, K. Mizushima, T. Fujimoto, T. Ogitsu, T. Obana, N. Amemiya, T. Orikasa, S. Takami, S. Takayama, and I. Watanabe, Design of a superconducting rotating gantry for heavy-ion therapy, Phys. Rev. ST Accel. Beams 15, 044701 (2012).

[5] Y. Iwata, T. Fujimoto, S. Matsuba, T. Fujita, S. Sato, T. Furukawa, Y. Hara, K. Mizushima, Y. Saraya, R. Tansho, N. Saotome, T. Shirai, and K. Noda, Beam commissioning of a superconducting rotating gantry for carbon-ion radiotherapy, Nucl. Instrum. Methods Phys. Res., Sect. A 834, 71 (2016).

[6] Y. Iwata, T. Fujita, T. Furukawa, T. Hara, S. Matsuba, K. Mizushima, T. Murakami, K. Noda, N. Saotome, Y. Saraya, S. Sato, T. Shirai, R. Tansho, T. Orikasa, S. Takayama, T. Fujimoto, T. Ogitsu, T. Obana, and N. Amemiya, Superconducting gantry for carbon-ion radiotherapy, in Proceedings of the 9th International Particle Accelerator Conference, IPAC 2018, Vancouver, 2018 (JACoW, Geneva, 2018), p. 1232.

[7] D. S. Robin, D. Arbelaez, S. Caspi, C. Sum, A. Sessler, W. Wan, and M. Yoon, Superconducting toroidal combinedfunction magnet for a compact ion beam cancer therapy gantry, Nucl. Instrum. Methods Phys. Res., Sect. A 659, 484 (2011). 
[8] S. Caspi, D. Arbelaez, H. Felice, R. Hafalia, D. Robin, C. Sun, W. Wan, and M. Yoon, Conceptual design of a $260 \mathrm{~mm}$ bore $5 \mathrm{~T}$ superconducting curved dipole magnet for a carbon beam therapy gantry, IEEE Trans. Appl. Supercond. 22, 4401204 (2012).

[9] J. Bokor and M. Pavlovic, An ion-optical design study of a carbon-ion rotating gantry with a superconducting final bending magnet, Nucl. Instrum. Methods Phys. Res., Sect. A 812, 122 (2016).

[10] Y. Iwata, T. Shirai, and K. Noda, Design of superconducting magnets for a compact carbon gantry, IEEE Trans. Appl. Supercond. 26, 4400104 (2016).

[11] E. Pedroni, D. Meer, C. Bula, S. Safai, and S. Zenklusen, Pencil beam characteristics of the next-generation proton scanning gantry of PSI: Design issues and initial commissioning results, Eur. Phys. J. Plus 126, 66 (2011).

[12] M. M. Necchi, S. Savazzi, C. Viviani, and J. Osorio Moreno, Functional specifications, union of light ion centers in Europe deliverable Report No. JRA6.1 2010, Vol. A565, p. 430, 2006.

[13] M. Pavlovic, E. Griesmayer, and R. Seemann, Beamtransport study of an isocentric rotating ion gantry with minimum number of quadrupoles, Nucl. Instrum. Methods Phys. Res., Sect. A 545, 412 (2005).

[14] OPERA version 15, http://www.cobham.com/.

[15] R. B. Meinke, C. L. Goodzeit, and M. J. Ball, Combined function magnets using double-helix coils, IEEE Trans. Appl. Supercond. 13, 1369 (2003).

[16] D. I. Meyer and R. Flasck, A new configuration for a dipole magnet for use in high energy physics applications, Nucl. Instrum. Methods 80, 339 (1970).

[17] S. Caspi, D. R. Dietderich, P. Ferracin, N. R. Finney, M. J. Fuery, S. A. Gourlay, and A. R. Hafalia, Design, fabrication, and test of a superconducting dipole magnet based on tilted solenoids, IEEE Trans. Appl. Supercond. 17, 2266 (2007).

[18] S. Caspi, L. N. Brouwer, T. Lipton, A. Hafalia, Jr., S. Prestemon, D. R. Dietderich, H. Felice, X. Wang, E. Rochepault, A. Godeke, S. Gourlay, and M. Marchevsky, Test results of CCT1A 2.4 T Canted-Cosine-Theta dipole magnet, IEEE Trans. Appl. Supercond. 25, 4002304 (2015).

[19] L. Bottura, A practical fit for the critical surface of NbTi, IEEE Trans. Appl. Supercond. 10, 1054 (2000).

[20] A. Devred, Practical low-temperature superconductors for electromagnets, CERN Yellow Report No. 2004-006, 2004.

[21] J. Park, G. Hahn, U. Bong, S. P. Kwon, H. Lee, M. Cho, J. Kim, Y. Chu, M. Y. Jung, K. J. Han, and S. Hahn, Conceptual design of a $5 \mathrm{~T} 200 \mathrm{~mm} 1 \mathrm{~T} / \mathrm{s}$ ramping $\mathrm{NbTi}$ synchrotron magnet for cancer treatment, IEEE Trans. Appl. Supercond., 29, 4004005 (2019).

[22] J. Kaugerts, G. Moritz, M. N. Wilson, A. Ghosh, A. den Ouden, I. Bogdanov, S. Kozub, P. Shcherbakov, L. Shirshov, L. Tkachenko, D. Richter, A. Verweij, G. Willering, P. Fabbricatore, and G. Volpini, Cable design for FAIR SIS 300, IEEE Trans. Appl. Supercond. 17, 1477 (2007).

[23] M. Yoon and D. S. Robin, Method of computing first-, second-, and third-order transfer coefficients for arbitrary fields, IEEE Trans. Nucl. Sci. 60, 3837 (2013).

[24] A. Dragt and J. Finn, Lie series and invariant functions for analytic symplectic maps, J. Math. Phys. (N.Y.) 17, 2215 (1976).

[25] Y. Li and X. Huang, A practical approach to extract symplectic transfer maps numerically for arbitrary magnetic elements, arXiv:1511.00710.

[26] G. Hoffstätter and M. Berz, Symplectic scaling of transfer maps including fringe fields, Phys. Rev. E 54, 5664 (1996). 